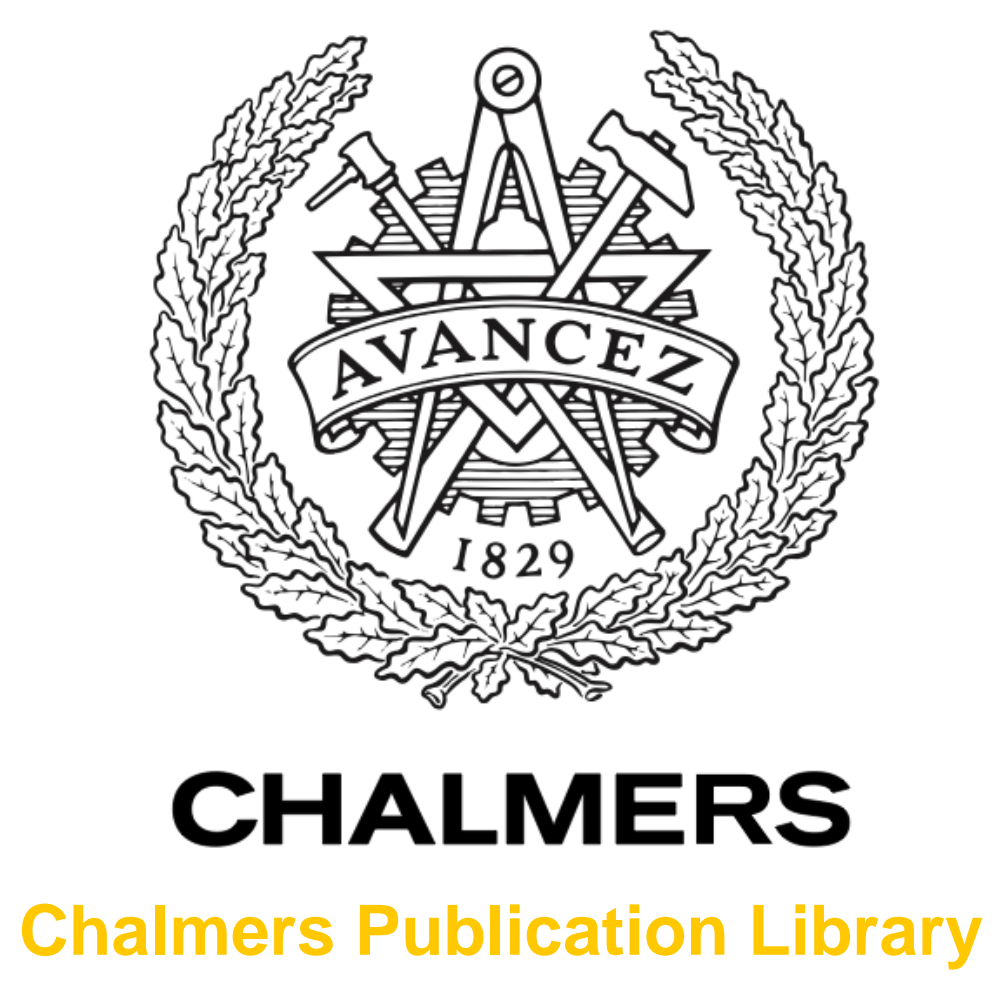

\title{
Diversity Gains in Random Line-Of-Sight and Rich Isotropic Multipath Environment
}

This document has been downloaded from Chalmers Publication Library $(\mathrm{CPL})$. It is the author's version of a work that was accepted for publication in:

\author{
2012 Loughborough Antennas \& Propagation Conference (LAPC), Lough-borough, \\ England, 12-13 Nov., 2012 \\ Citation for the published paper: \\ Carlsson, J. ; Carlberg, U. ; Kildal, P. (2012) "Diversity Gains in Random Line-Of-Sight and \\ Rich Isotropic Multipath Environment". 2012 Loughborough Antennas \& Propagation \\ Conference (LAPC), Lough-borough, England, 12-13 Nov., 2012
}

http://dx.doi.org/10.1109/LAPC.2012.6402936

Downloaded from: http://publications.lib.chalmers.se/publication/166926

Notice: Changes introduced as a result of publishing processes such as copy-editing and formatting may not be reflected in this document. For a definitive version of this work, please refer to the published source. Please note that access to the published version might require a subscription. 


\section{Diversity Gains in Random Line-Of-Sight and Rich Isotropic Multipath Environment}

\author{
Jan Carlsson, Ulf Carlberg \\ Department of Electronics \\ SP Technical Research Institute of Sweden \\ Borås, Sweden \\ jan.carlsson@sp.se, ulf.carlberg@sp.se
}

\author{
Per-Simon Kildal \\ Department of Signals and Systems \\ Chalmers University of Technology \\ Gothenburg, Sweden \\ per-simon.kildal@chalmers.se
}

\begin{abstract}
Antenna diversity gain for theoretical as well as measured antennas is studied in two extreme environments, the rich isotropic multipath environment (RIMP) and the random line-of-sight environment. The RIMP diversity gain was previously defined based on improved fading performance, here we equivalently consider it as a metric for the cumulative improvement of the $1 \%$ worst users randomly distributed in the RIMP environment. Similarly, we consider the diversity gain in the random line-of-sight environment to be the performance improvement of the $1 \%$ of the users which receives the weakest signal relative to a theoretical Rayleigh distribution of the signal levels among the users. The random line-of-sight environment is regarded as being caused by the statistics of an ensemble of users (or terminals) with arbitrary 3D orientations.
\end{abstract}

Keywords-MIMO; antenna diversity gain; random line-ofsight; OTA measurements

\section{INTRODUCTION}

Modern wireless devices with multiport antennas are able to exploit the multipath environment by the use of multipleinput multiple-output (MIMO), diversity and spatial multiplexing. Currently there are a lot of work in different standardization groups for the development of new test methods that can characterize also the antenna diversity and spatial multiplexing. These methods are commonly referred to as over-the-air (OTA) tests in contrast to conductive tests where the antennas are bypassed and a multipath emulator is connected directly to the input of the transmitter/receiver.

The statistical multipath environments in urban areas and indoor are normally rich and close to isotropic, where the latter means that the angles-of-arrivals (AoA) of the incident waves on the wireless device are uniformly distributed over all directions in 3D space. The antenna diversity gain is properly defined in the rich isotropic multipath environment (RIMP) [1] and can conveniently be measured in a reverberation chamber that properly emulates the RIMP [2]. Although the RIMP can be considered to be an extreme environment it is a proper reference environment for mobile wireless devices if we also take the arbitrary orientation of the device into account. However, since not all environments are rich and close to isotropic, we study in this paper also another extreme environment, the random line-of-sight environment.

This work was supported in part by the Swedish Governmental Agency for Innovation Systems (VINNOVA) within the VINN Excellence Center Chase at Chalmers University of Technology.
In rural areas and close to the base station there will often be a significant line-of-sight (LOS) component, i.e. a dominant wave incident from a specific direction. This is a classical environment present in all point-to-point communication systems with fixed antenna installations. However, when considering mobile communication systems the orientations of the user as well as the mobile device are arbitrary and therefore we will in the present paper study antenna diversity for an ensemble of users in the LOS environment. We follow the definitions in [3] and consider the so-called user-distributed 3D-random LOS environment. This can be described as a single incident wave with constant amplitude and with arbitrary incidence direction and polarization. We study the antenna diversity by studying an ensemble of users having the same device. Other publications related to random LOS like [4-6] have considered moving single users, whereas we are concerned about evaluating performance over an ensemble of users. It should be pointed out that the observed cumulative distribution functions (CDFs) in our case are not representing fading, i.e. a time-varying signal, but rather a spatial distribution over an ensemble of users having the same device.

The CDFs for multiport antennas in user-distributed 3Drandom LOS can be computed with ViRM-Lab, a computer code for analyzing performance of wireless terminals in multipath with many arbitrary incident waves [7] based on standard receive antenna equivalent circuits [8]. However, since we here only need to consider one incident wave we simplify the analysis by uniformly sampling the 3D far-field function over the unit sphere, and then create the CDF from these samples. This is computationally very fast and we have verified the results by comparison with the more general ViRM-Lab.

\section{CuMUlative DiVERSITY GAIN IN RiCH ISOTROPIC} MULTIPATH AND RANDOM LINE-OF-SIGHT ENVIRONMENTS

Diversity and MIMO antennas have multiple ports and their performance can be quantified by processing the wireless channel between the ports on both sides of the communication link. Previous papers such as [2] have defined the diversity gain from the CDFs in a RIMP environment by considering a moving user. The CDFs are then generated by collecting time samples of the received signals on each port, as well as of the diversity combined port. In addition a CDF is generated from a 
reference antenna with known efficiency (usually assumed to be $0 \mathrm{~dB}$ ) in the same RIMP environment. The average received power from this reference antenna is used to normalize the other CDFs. All single-port CDFs will follow the theoretical Rayleigh distribution independent of the directivity of the antenna provided the environment is rich enough, but will be shifted towards lower received power levels relative to the reference $\mathrm{CDF}$ by an amount equal to the total embedded radiation efficiency seen at the port [2]. Previously, diversity gain was defined for RIMP based on improved fading performance at a certain CDF level, often taken to be $1 \%$. However, we could just as well consider it to be a metric of the cumulative improvement of the $1 \%$ worst users randomly distributed in the RIMP environment as is argued for in [9]. We then refer to it as the cumulative RIMP diversity gain and measure it relative the theoretical Rayleigh distribution. We choose in our examples maximum ratio combining (MRC) of the voltages at each port as the diversity scheme and we consider only two port antennas. Of convenience, we neglect the embedded element efficiencies by assuming lossless matched antennas, as well as no coupling loss between the antenna ports. We can do this without loss of generality since efficiency will simply shift the CDFs and not change their shapes as discussed above.

For the user-distributed 3D-random LOS environment we consider an ensemble of users. If we have a large enough ensemble, it is easy to understand that the AoA of the LOS will be uniformly distributed in the azimuthal plane since each user can have any azimuthal orientation. It is not so obvious that the AoA will be uniformly distributed also in the elevation plane, as argued for in [9]. A related interchanging of horizontal and vertical directions appears also in modern handheld tablets that can be used for any orientation of the display, i.e. any of its four edges can be pointing up, as shown in Fig. 1. Thus, we can defend studying a user-distributed 3D-random LOS.

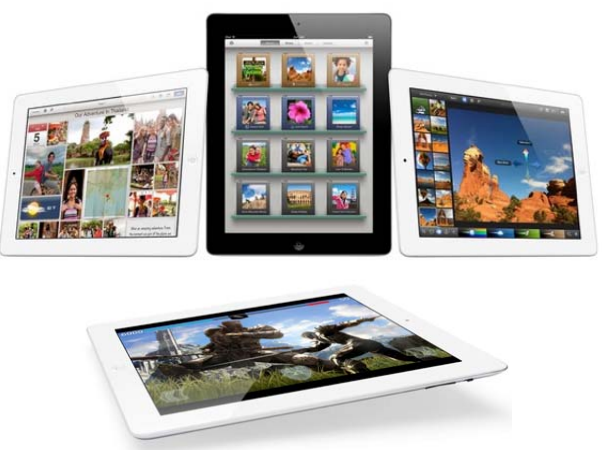

Figure 1. Modern tablets can be used for any orientation relative to the surrounding environment. Their built-in antennas will therefore experience any incident wave (LOS) as being uniformly distributed over all AoA, i.e. a 3D-random LOS.

For the random LOS environment we follow a similar approach for generating the CDF curves as we did for the RIMP environment. The difference is that we now generate our voltage samples by considering each user, one at a time, instead of considering a moving user. Since each user has a random $3 \mathrm{D}$ orientation and the exciting single wave comes from a specific direction the CDFs are simply generated by sampling the embedded far-field functions over the unit sphere and arranging the samples from lowest to highest levels.

As an example we consider the mockup of a mobile phone with a two port diversity antenna shown in Fig. 2. The CDFs for the RIMP and user-distributed 3D-random LOS environments together with the MRC combined CDFs are shown in Fig. 3. These results are based on measured embedded far-field functions, i.e. far-field functions measured on each port with the other port terminated in $50 \mathrm{ohm}$. For the calculations $10^{5}$ realizations were used for both environments, and twenty linearly polarized waves were used in each realization for the RIMP and one for the random LOS environment. In the figure the CDFs are normalized to the highest average received power among the two ports which means that the total embedded element efficiency for the strongest port do not show up in the curves and therefore one of the RIMP CDFs coincide with the theoretical Rayleigh. This can be seen at low CDF-levels were the calculated curve has not fully converged. In order to be able to compare CDFs and diversity gains for different antennas we need to correct for the efficiencies, for the considered handheld position and frequency these were $-7.1 \mathrm{~dB}$ and $-8.9 \mathrm{~dB}$ for port 1 and port 2 , respectively.

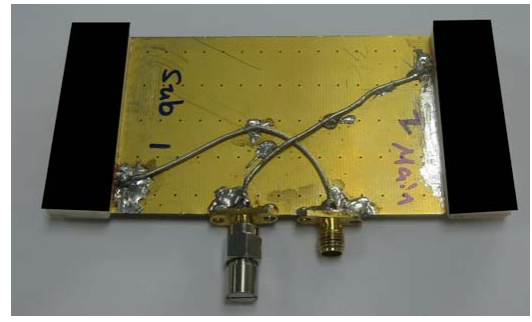

Figure 2. Photo of mobile phone mockup with two port diversity antenna (masked antenna elements). The size is $115 \times 55 \times 12 \mathrm{~mm}^{3}$.

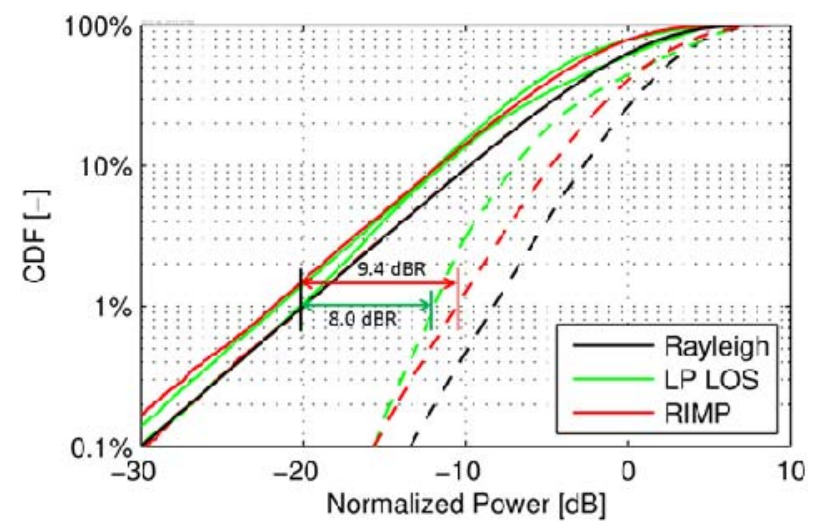

Figure 3. Apparent cumulative RIMP diversity gain $(9.4 \mathrm{dBR})$ and 3Drandom LOS diversity gain $(8.0 \mathrm{dBR})$ for the two port antenna shown in Fig. 2 in handheld position. Solid lines represent the CDFs on each port for the two environments. Dashed lines represent the maximum ratio combined cases. The corresponding Rayleigh cases are also shown. LP LOS means linearly polarized random LOS environment.

In Fig. 3 we see that the RIMP CDFs follow the theoretical Rayleigh as expected, one of them coincide exactly with the Rayleigh curve due to the normalization and the other is shifted with the difference in embedded element efficiencies between 
the two ports. We can also see that the 3D-random LOS CDFs are close to being Rayleigh distributed, but this is not necessarily always the case, as will be seen later in the paper. The cumulative diversity gains relative to the theoretical Rayleigh at the $1 \%$ level are also shown in the figure for the two environments. The unit is $\mathrm{dBR}$ which means $\mathrm{dB}$ relative to Rayleigh. It should be pointed out that the user-distributed cumulative diversity gain in the 3D-random LOS environment is a measure of the performance of a large group of users in the environment. Thus, a diversity gain of $9.4 \mathrm{dBR}$ means that the $1 \%$ of the users which receives the weakest signal will experience a performance improvement of $9.4 \mathrm{~dB}$ or more relative to a theoretical Rayleigh distribution of the signal levels among the users.

\section{CDFS AND DIVERSITY GAINS OF ELEMENTARY SMALL ANTENNAS IN 3D-RANDOM LOS ENVIRONMENT}

Practical small antennas may in free space radiate similar to small electric or magnetic dipoles, or linear combinations of them. We will therefore show some results for such elementary antennas even if we know that the chassis of the device and the influence of the user will have a large impact and make them radiate differently in practice. Our choice in this section is to study the Huygens source which is a linear combination of elementary electric and magnetic dipoles.
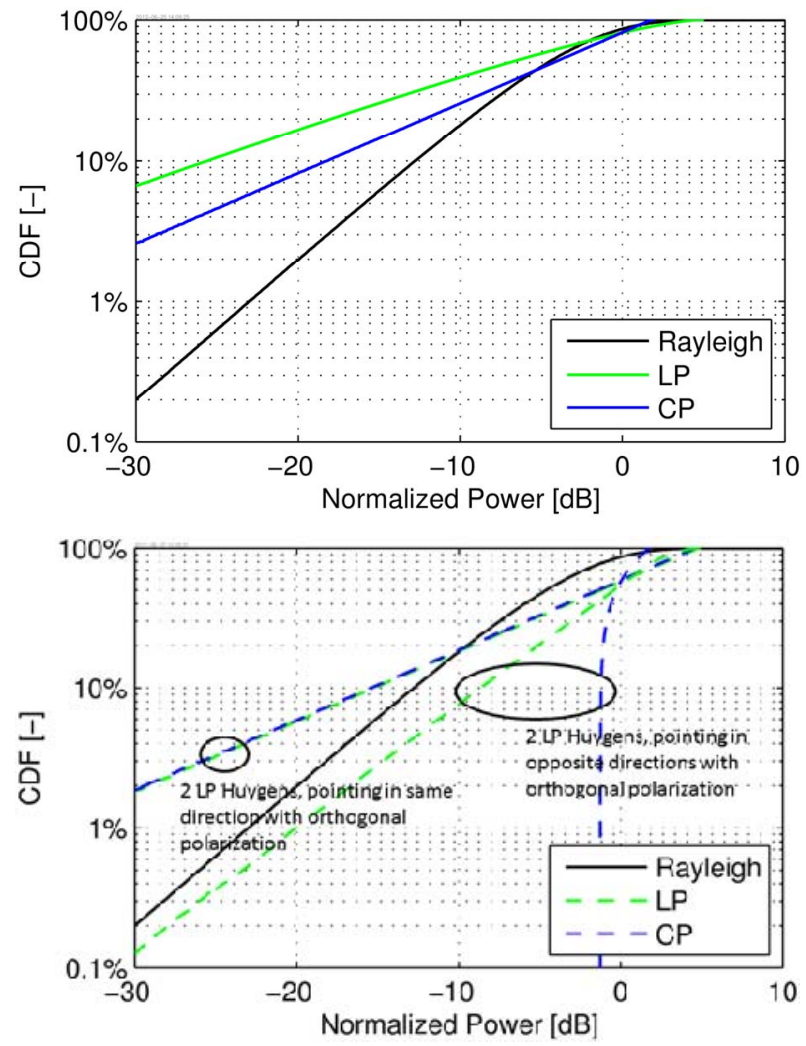

Figure 4. CDF (solid) and MRC diversity combined (dashed) curves in userdistributed 3D-random LOS environment with linear (green) and circular (blue) polarizations. Rayleigh curves are included for reference. Upper: Single linearly polarized Huygens source. Lower: Two combined Huygens sources, pointing in the same direction with orthogonal polarizations and pointing in opposite directions with orthogonal polarizations, respectively.
The Huygens source has a far-field function of the form $1+\cos (\theta)$ giving a directivity of $4.8 \mathrm{dBi}$. This is the highest directivity available among free space small antennas. The CDFs and MRC combined curves for a single Huygens source are shown in the upper graph in Fig. 4 and those of two differently combined Huygens sources in the lower graph. We see that the CDF of a single Huygens source in a linearly polarized (LP) 3D-random LOS is worse than the theoretical Rayleigh, whereas the same Huygens source for the circularly polarized (CP) 3D-random LOS is much better. Even though not visible in the figure, the difference between the two cases at the $1 \%$ CDF-level is $10.8 \mathrm{~dB}$. This implies that since terminals usually are linearly polarized it would be beneficial to use circular polarized base stations. For the case of two Huygens sources pointing in the same directions but with orthogonal polarizations we see that there is no difference between the cases of linearly polarized and circularly polarized 3D-random LOS. However, when the Huygens sources are pointing in opposite directions and have orthogonal polarizations there is a much better reception. In this case there is also a dramatic difference between the two considered polarizations of the 3Drandom LOS. We see that for circularly polarized 3D-random LOS the cumulative MRC diversity gain relative to the theoretical Rayleigh at the $1 \%$ level is $21.7 \mathrm{dBR}$. This illustrates the importance of having far-fields that covers most directions of the $3 \mathrm{D}$ sphere to minimize probability of low received power.

\section{CDFs AND DIVERSITY GAINS OF MOBILE PHONE MOCKUP WITH DIVERSITY ANTENNA IN THE 3D-RANDOM LOS ENVIRONMENT}

As an example of a practical antenna we study the mobile phone mockup with a two-port diversity antenna shown in Fig. 2. We use the measured complex valued embedded far-field functions for each port for the case when the mockup is held by a human hand phantom and calculates the CDFs as described earlier. The CDFs for one of the ports for 22 uniformly distributed frequencies in the range $750-960 \mathrm{MHz}$ are shown in the upper graph in Fig. 5 and the corresponding MRC diversity combined CDFs in the lower graph.

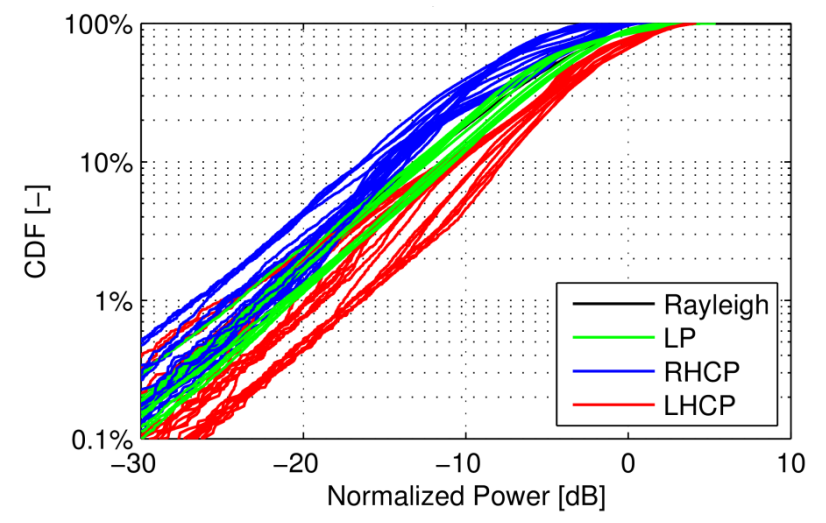




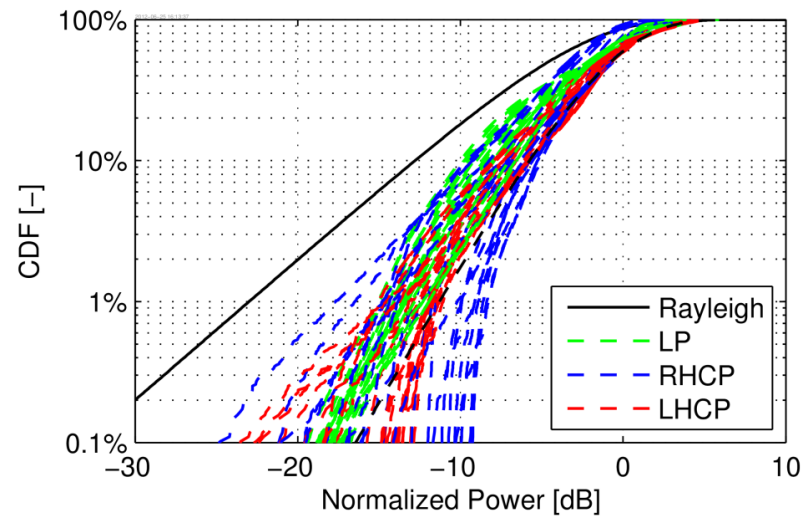

Figure 5. Upper: CDF curves for one port on the diversity antenn in Fig. 2 for 22 frequencies between 750 and $960 \mathrm{MHz}$ for linear, right-hand circular and left-hand circular polarized 3D-random LOS, respectively. Lower: corresponding MRC diversity combined CDF curves.

In Fig. 5 the statistics of the received power for LP, RHCP and LHCP 3D-random LOS environments are shown as curves with different colors. In the upper part of the figure we see that the CDF curves form a bundle around the theoretical Rayleigh, and the green LP curves are in-between the circular polarized cases. This indicates that the embedded far-field function for this particular antenna port is mostly left hand circularly polarized. The MRC diversity combined CDFs are shown in the lower part of Fig. 5 where it can be noted that we have a positive $\mathrm{dBR}$ value for all cases. The deviations of the $\mathrm{CDF}$ curves from Rayleigh at the $1 \%$ level are shown in Fig. 6, for both single ports and after MRC diversity combinations as a function of frequency.

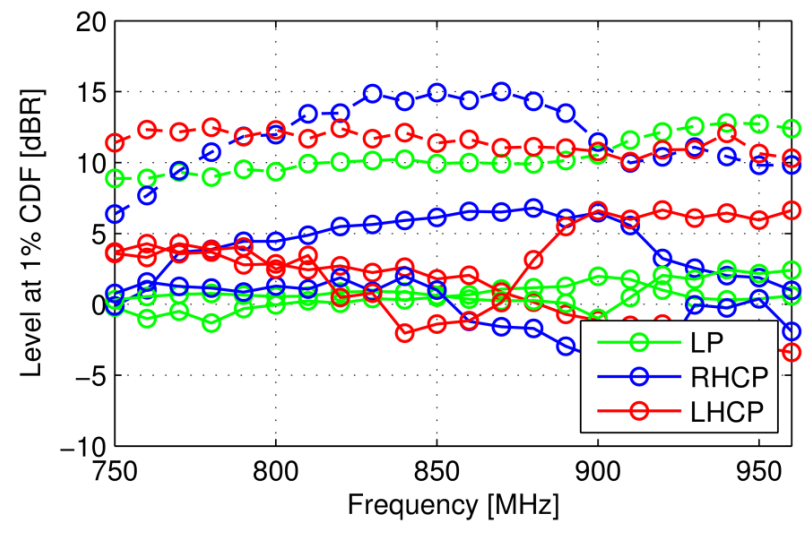

Figure 6. Cumulative user-distributed performance at $1 \% 1 \%$ CDF level in $\mathrm{dBR}$ as a function of frequency for CDFs in Fig. 5. Uncombined CDF levels (solid) on both ports and for different polarizations of the 3D-random LOS, and MRC curves (dashed).

\section{CONCLUSIONS}

We have studied the statistics of many users in the 3Drandom LOS environment and compared it to the RIMP environment. Various antennas (theoretical Huygens sources and measured far-fields from a mockup) were considered in order to evaluate these environments. In the RIMP environment only the efficiency of antennas matters and the statistics of received power will always follow the theoretical Rayleigh curve. In the 3D-random LOS environment we have seen that it is in addition important to have far-fields that are close to omni-directional, i.e. radiates in most directions of the 3D sphere. Also for linear polarized terminal antennas it is beneficial with circular polarized base stations.

\section{ACKNOWLEDGMENT}

The authors are grateful to Thomas Bolin and Zhinong Ying at Sony Ericsson Mobile Communications, Lund, Sweden for providing the mockup and the measured far-field functions.

\section{REFERENCES}

[1] P. S. Kildal, K. Rosengren, B. Joonho, and L. Juhyung, "Definition of effective diversity gain and how to measure it in a reverberation chamber," Microwave and Optical Technology Letters, vol. 34, pp. 56-9, 2002.

[2] P. S. Kildal and K. Rosengren, "Correlation and capacity of MIMO systems and mutual coupling, radiation efficiency, and diversity gain of their antennas: simulations and measurements in a reverberation chamber," IEEE Communications Magazine, vol. 42, pp. 104-112, 2004.

[3] P. S. Kildal, U. Carlberg, and J. Carlsson, "Definition of Antenna Diversity Gain in User-Distributed 3D-Random Line-Of-Sight," submitted to IEEE Antennas Wireless Propag. Lett., Feb. 2012.

[4] G. Alfano and A. De Maio, "Sum of Squared Shadowed-Rice Random Variables and its Application to Communication Systems Performance Prediction," IEEE Trans. Wireless Communications, vol. 6, pp. 35403545, 2007.

[5] M. Godavarti, T. L. Marzetta, and S. Shamai, "Capacity of a mobile multiple-antenna wireless link with isotropically random Rician fading," IEEE Trans. Information Theory, vol. 49, pp. 3330-3334, 2003.

[6] A. Sibille and M. A. Mellah, "A statistical model of handsets effective gain accounting for user influence and local propagation," in Proceedings of the Fourth European Conference on Antennas and Propagation (EuCAP 2010), 2010, pp. 1-4.

[7] U. Carlberg, J. Carlsson, A. Hussain, and P. S. Kildal, "Ray based multipath simulation tool for studying convergence and estimating ergodic capacity and diversity gain for antennas with given far-field functions," in $20^{\text {th }}$ Internat. Conf. Applied Electromagnetics and Communications (ICECom), 20-23 Sept. 2010, Dubrovnik, Kroatia, 2010.

[8] P. S. Kildal, "Equivalent circuits of receive antennas in signal processing arrays," Microwave and Optical Technology Letters, vol. 21, pp. 244246, 1999.

[9] P.-S. Kildal, C. Orlenius, and J. Carlsson, "OTA Testing in Multipath of Antennas and Wireless Devices with MIMO and OFDM," to appear in Proceedings of IEEE, July 2012 